\title{
216 MULTIPLEX BASE EDITING OF NK CELL TO ENHANCE CANCER IMMUNOTHERAPY
}

Minjing Wang*, Mitchell Kluesner, Patricia Claudio Vázquez, Beau Webber, Branden Moriarity. University of Minnesota, Minneapolis, MN, USA

Background Natural killer (NK) cells have many unique features that have gained attention in cancer immunotherapy. NK cells can kill in antigen independent and dependent fashion, can be used as an allogeneic product, and perform antibodydependent cell-mediated cytotoxicity (ADCC). However, NK cell function is regulated by many activating and inhibitory receptors, which cancer cells take advantage of to avoid being killed by NK cells. NK cells are also known for their technical and biological challenges which result in low editing efficiencies, compared to $\mathrm{T}$ cells and other immune cells.

Methods Base editing (BE) is a CRISPR-Cas9 based genome editing technology that allows precise single base transitions. Previously, we reported a high efficiency method for multiplex engineering of $\mathrm{T}$ cells using $\mathrm{BE}$ and thus reasoned that applying similar concepts in NK cells may offer an opportunity to alter many genes simultaneously at higher efficiency through multiplex base editing. We thus selected a panel of genes bearing critical roles in NK cell function for immunotherapy, including inhibitory intracellular regulator $\mathrm{AHR}$ and $\mathrm{CISH}$, inhibitory checkpoint receptor KLRG1, TIGIT, KLRC1, and PDCD1, and Fc receptor CD16A. CD16A is responsible for NK cell ADCC and is regulated via cleavage upon NK activation. Non-cleavable CD16A improves ADCC killing and can be achieved through single-base substitution with $\mathrm{BE}$.

Results Using the adenosine BE (ABE8e), we achieved multiplex editing (6 genes) rates up to $99 \%$ and $95 \%$ editing/ knockout at DNA and protein levels, respectively. Notably, we assessed for reduction in editing efficiency when additional genes were targeted and found no significant reduction in editing efficiencies when targeting up to 6 genes simultaneously. Moreover, functional evaluation of non-cleavable CD16A NK cells revealed up to $35 \%$ increase of cytotoxicity against Raji cells.

Conclusions We were able to achieve high multiplex editing efficiency in primary human NK cells using ABE8eand there was no significant decrease of editing efficiency as the number of gene of interest increases, up to 6 genes in total. Functional assay confirmed increased $\mathrm{NK}$ cell cytotoxicity against tumor cells. Our end goal is to achieve high efficiency multiplex editing in CAR-expressing NK cells to further improve NK cell activity and toxicity for cancer immunotherapy.

\section{REFERENCE}

1.. Webber $B$, Lonetree $C$, Kluesner $M$, et al. Highly efficient multiplex human $T$ cell engineering without double-strand breaks usingCas9 base editors. Nat Commun 2019;10:5222.

http://dx.doi.org/10.1136/jitc-2021-SITC2021.216 